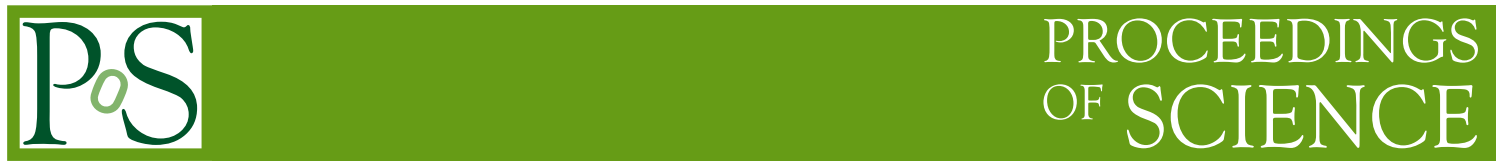

\title{
Particle Production and Deconfinement Threshold
}

\author{
Johann Rafelski* \\ University of Arizona, Tucson, AZ 85721, USA and \\ Department für Physik der Ludwig-Maximilians-Universität München, \\ Maier-Leibnitz-Laboratorium, Am Coulombwall 1, 85748 Garching, Germany \\ E-mail: Rafelski@Physics.Arizona.EDU
}

\section{Jean Letessier}

LPTHE, Université Paris 7, 2 place Jussieu, F-75251 Cedex 05

We present a detailed analysis of the NA49 experimental particle yield results, and discuss the physical properties of the particle source. We explain in depth how our analysis differs from the work of other groups, what advance this implies in terms of our understanding, and what new physics about the deconfined particle source this allows us to recognize. We answer several frequently asked questions, presenting a transcript of a discussion regarding our data analysis. We show that the final NA49 data at 40,80,158 AGeV lead to a remarkably constant extensive thermal chemical-freeze-out properties of the fireball. We discuss briefly the importance of thermal hadronization pressure.

8th Conference Quark Confinement and the Hadron Spectrum

September 1-6, 2008

Mainz. Germany

\footnotetext{
${ }^{*}$ Speaker.
} 


\section{Deconfinement and Hadron Production}

Enhanced production of strange hadrons, and of (strange) antibaryons is a signature of quarkgluon plasma (QGP) formation in relativistic heavy ion collisions [1]. We illustrate on left in figure 1 the two step mechanism of hadron production from quark-gluon matter: upon deconfinement thermal glue emerges from parton matter, thermal gluon fusion reactions produce quark pairs in mass range $m_{i}<3 T$ [2]. Subsequently, at a later time and thus at a much lower ambient temperature, quarks merge into final state hadrons $[3,4]$. On the right in figure 1 we show the relative yield as function of $p_{\perp}$ of baryon to meson abundance which are quite different from what is found in $p p$ reactions [5]. This confirms that the relativistic heavy ion hadron production differs from the string breaking mechanism of hadron production [6]. This result found also at top SPS energy range [7].
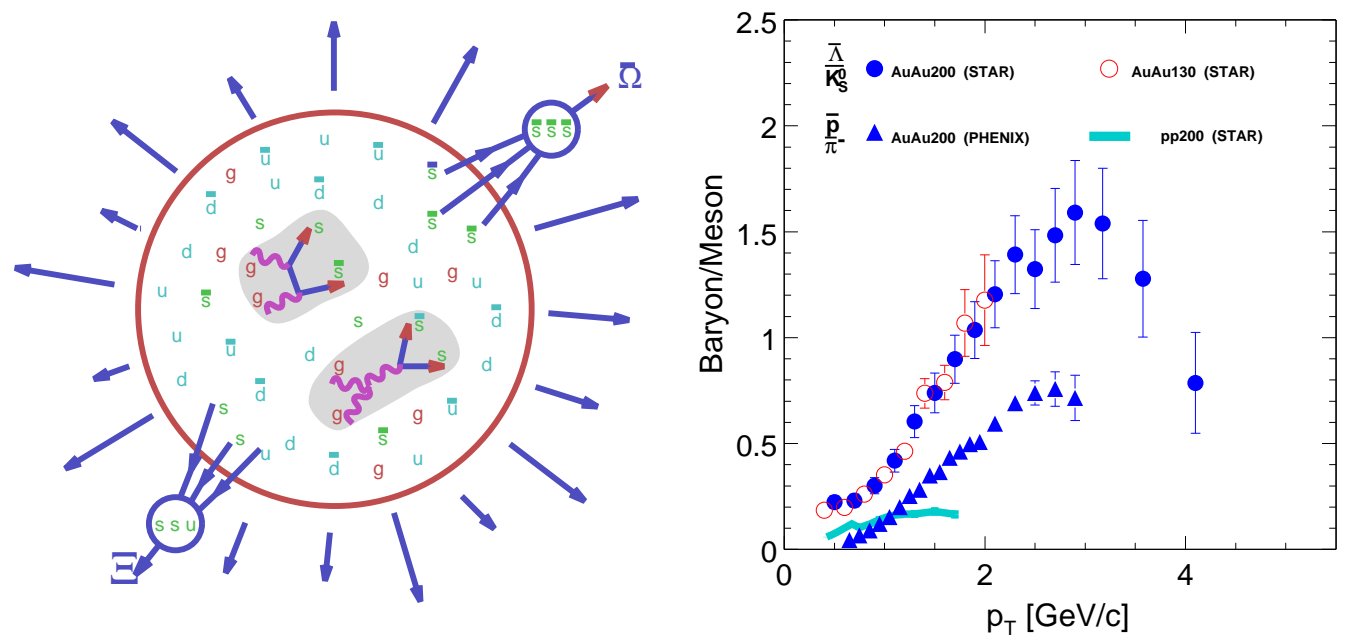

Figure 1: On left, qualitative illustration of (strange) hadron production in QGP: gluon fusion reactions populate flavor yield, subsequently at phase breakup, quarks merge into final state hadrons. On right, example of baryon to meson ratio experimental results $\bar{\Lambda} / K_{S}$ and $\bar{p} / \pi$ in Au-Au compared to $p p$ collisions as a function of $p_{\perp}$.

Of particular relevance in this work is the parameter controlling the absolute yield of quark pairs $\gamma_{q}^{\mathrm{QGP}}$ for $u, d$ and $\gamma_{s}^{\mathrm{QGP}}$ for $s$ (and $\gamma_{c}^{\mathrm{QGP}}, \gamma_{b}^{\mathrm{QGP}}$ for $c, b$ not further discussed here). In an equilibrating system all $\gamma_{i}^{\mathrm{QGP}} \rightarrow 1$ as function of time, one can argue that at RHIC strange flavor in QGP is nearly equilibrated. However, in an experiment, we observe hadrons, these yields are subject to different parameters operating in the hadron phase (HP) with the initial values $\gamma_{i}^{\mathrm{HP}}\left(t_{f}\right)$ If hadrons do not scatter much after formation, as is the case in the single freeze-out model $[8,9]$, there is little if any change of $\gamma_{i}^{\mathrm{HP}}\left(t_{f}\right)$.

Remarkably, these two particle yield parameters, $\gamma_{q}^{\mathrm{HP}}\left(t_{f}\right)$ and $\gamma_{s}^{\mathrm{HP}}\left(t_{f}\right)$, also control the relative yield of baryons to mesons shown on right in figure 1,

$$
\frac{\text { baryons }}{\text { mesons }} \propto \frac{\gamma_{q}^{\mathrm{HP} 3}}{\gamma_{q}^{\mathrm{HP} 2}} \cdot\left(\frac{\gamma_{s}^{\mathrm{HP}}}{\gamma_{q}^{\mathrm{HP}}}\right)^{n} \propto \gamma_{q}^{\mathrm{HP}},
$$

where $\gamma_{s} / \gamma_{q}$ shifts the yield of strange vs non-strange hadrons, thus for example the relative yields 
of hadrons obey:

$$
\frac{\bar{\Lambda}(\bar{u} \bar{d} \bar{s})}{\bar{p}(\bar{u} \bar{u} \bar{d})} \propto \frac{\gamma_{s}^{\mathrm{HP}}}{\gamma_{q}^{\mathrm{HP}}}, \quad \frac{\mathrm{K}^{+}(u \bar{s})}{\pi^{+}(u \bar{d})} \propto \frac{\gamma_{s}^{\mathrm{HP}}}{\gamma_{q}^{\mathrm{HP}}}, \quad \frac{\phi}{h} \propto \frac{\gamma_{s}^{2 \mathrm{HP}}}{\gamma_{q}^{2 \mathrm{HP}}}, \quad \frac{\Omega(s s s)}{\Lambda(s u d)} \propto \frac{\gamma_{s}^{2 \mathrm{HP}}}{\gamma_{q}^{2 \mathrm{HP}}} .
$$

The above observation, when combined with the results shown on right in figure 1, imply that the baryon to meson yield and thus $\gamma_{q}^{\mathrm{HP}}\left(t_{f}\right)$ is arising from microscopic dynamics of hadronization. This establishes the necessity to include the occupancy parameters in order to describe the yields of hadrons, since this is the parameter which allows for a reaction dependent relative yield of mesons and baryons. Conversely, a study of particle yields with a fixed value $\gamma_{q}^{\mathrm{HP}}=1$ presumes certain relative yield of baryons to mesons and this is over-constraining a model of hadronization. Therefore, in such an approach, in order to describe the data precisely, the control of the relative number of produced mesons and baryon is achieved by manipulating the hadron spectrum [10] including additional meson and baryon resonances, it is possible to fine tune the final relative yield of meson and baryons. Naturally, such a fine tuning of the hadron mass spectrum in fact confirms the failure of the 'equilibrium' model [11], and confirms the necessity to use the non-equilibrium occupancy parameters, if not for any fundamental reason, than in order to fudge the errors in the mass spectrum of hadrons.

The greatest experimental sensitivity of the baryon to meson ratio is present when the respective yields are comparable, such that the particle yields are a sensitive measure of this ratio. This is the case for the intermediate energy range at SPS, e.g., in the $80 \mathrm{AGeV} \mathrm{Pb}$ collisions with laboratory fixed target. Our choice to consider the SPS energy range in this presentation is in part dictated by this observation, and in part by the fact that we expect a change in reaction mechanism at an energy range below the RHIC 'high' energy runs.

Other statistical hadronization (SH) parameters we derive from the data are the source volume $V$ and the temperature $T$, at which particles stop changing in yield (chemical freeze-out). Moreover, we obtain chemical potentials $\mu_{B}=3 \mu_{q}=3 T \ln \lambda_{q}, \mu_{S}=T \ln \left(\lambda_{q} / \lambda_{s}\right)$, related to conserved quantum numbers: baryon number and strangeness, respectively. We also obtain $\lambda_{I 3}$ which allows to conserve the net charge and expresses the asymmetry in the 3-rd component of the isospin. Especially for low energy reactions where the particle yield is relatively low this parameter differs significantly from unity.

The particle yields measure the values of SH parameters assuming that all particles stop changing their abundance nearly at the same point in time. Here, the yield of stable hadronic particles (ignoring weak decays) includes hadronic resonance decay chain. Note that even if hadronic resonances continue to react and their number changes in time after QGP breakup, the final number of stable particles is unchanged. Thus, the chemical freeze-out values of SH parameters, and the associated physical properties of the system at hadronization can be related to the QGP breakup condition, if these hadrons are free-streaming out of the deconfined fireball.

Since the phase space density is in general different in the two phases, in order to preserve entropy (the valance quark pair number) across the phase boundary there must be a jump in the phase space occupancy parameters $\gamma_{q}$ - this effect replaces the increase in volume in a slow re-equilibration with mixed phase which accommodates transformation of a QGP entropy dense phase into HP dilute phase. Similarly, there is a jump in strangeness occupancy since QGP is a more strangeness dense phase than is HP. $\gamma_{i}^{\mathrm{HP}}\left(t_{f}\right)>\gamma_{i}^{\mathrm{QGP}}\left(t_{f}\right), i=q$, s, again due to rather large 
Table 1: AGS (on left) and SPS energy range particle multiplicity data sets used in fits (see text). In bottom of table, we show the fitted statistical parameters and the corresponding chemical potentials.

\begin{tabular}{|l|c|ccccc|}
\hline $\mathrm{E}[A \mathrm{AGeV}]$ & 11.6 & 20 & 30 & 40 & 80 & 158 \\
$\sqrt{s_{\mathrm{NN}}}[\mathrm{GeV}]$ & 4.84 & 6.26 & 7.61 & 8.76 & 12.32 & 17.27 \\
$y_{\mathrm{CM}}$ & 1.6 & 1.88 & 2.08 & 2.22 & 2.57 & 2.91 \\
$N_{4 \pi}$ centrality & most central & $7 \%$ & $7 \%$ & $7 \%$ & $7 \%$ & $5 \%$ \\
\hline$N_{W}, \mathrm{AGS}: p / \pi^{+}$ & $1.23 \pm 0.13$ & $349 \pm 6$ & $349 \pm 6$ & $349 \pm 6$ & $349 \pm 6$ & $362 \pm 6$ \\
$Q / b$ & $0.39 \pm 0.02$ & $0.394 \pm 0.02$ & $0.394 \pm 0.02$ & $0.394 \pm 0.02$ & $0.394 \pm 0.02$ & $0.39 \pm 0.02$ \\
$(s-\bar{s}) /(s+\bar{s})$ & $0 \pm 0.05$ & $0 \pm 0.05$ & $0 \pm 0.05$ & $0 \pm 0.05$ & $0 \pm 0.05$ & $0 \pm 0.05$ \\
\hline$\pi^{+}$ & $133.7 \pm 9.9$ & $190.0 \pm 10.0$ & $241 \pm 13$ & $293 \pm 18$ & $446 \pm 27$ & $619 \pm 48$ \\
$\pi^{-}, \mathrm{AGS}: \pi^{-} / \pi^{+}$ & $1.23 \pm 0.07$ & $221.0 \pm 12.0$ & $274 \pm 15$ & $322 \pm 19$ & $474 \pm 28$ & $639 \pm 48$ \\
$\mathrm{~K}^{+}, \mathrm{AGS}: \mathrm{K}^{+} / \mathrm{K}^{-}$ & $5.23 \pm 0.5$ & $40.7 \pm 2.9$ & $52.9 \pm 4.2$ & $56.1 \pm 4.9$ & $73.4 \pm 6$ & $103 \pm 10$ \\
$\mathrm{~K}^{-}$ & $3.76 \pm 0.47$ & $10.3 \pm 0.3$ & $16 \pm 0.6$ & $19.2 \pm 1.5$ & $32.4 \pm 2.2$ & $51.9 \pm 4.9$ \\
$\phi, \mathrm{AGS}: \phi / \mathrm{K}^{+}$ & $0.025 \pm 0.006$ & $1.89 \pm 0.53$ & $1.84 \pm 0.51$ & $2.55 \pm 0.36$ & $4.04 \pm 0.5$ & $8.46 \pm 0.71$ \\
$\Lambda$ & $18.1 \pm 1.9$ & $27.1 \pm 2.4$ & $36.9 \pm 3.6$ & $43.1 \pm 4.7$ & $50.1 \pm 10$ & $44.9 \pm 8.9$ \\
$\bar{\Lambda}$ & $0.017 \pm 0.005$ & $0.16 \pm 0.05$ & $0.39 \pm 0.06$ & $0.68 \pm 0.1$ & $1.82 \pm 0.36$ & $3.68 \pm 0.55$ \\
$\Xi^{-}$ & & $1.5 \pm 0.3$ & $2.42 \pm 0.48$ & $2.96 \pm 0.56$ & $3.8 \pm 0.87$ & $4.5 \pm 0.20$ \\
$\bar{\Xi}$ & & & $0.12 \pm 0.05$ & $0.13 \pm 0.03$ & $0.58 \pm 0.19$ & $0.83 \pm 0.04$ \\
$\Omega+\bar{\Omega}$, or $\mathrm{K}_{\mathrm{S}}$ & & & & $0.14 \pm 0.07$ & & $81 \pm 4$ \\
\hline$V\left[\mathrm{fm}{ }^{3}\right]$ & & & & & & \\
$T[\mathrm{MeV}]$ & $153.5 \pm 0.8$ & $151.7 \pm 2.8$ & $123.8 \pm 3$ & $130.9 \pm 4.4$ & $135.2 \pm 0.01$ & $136.0 \pm 0.01$ \\
$\lambda_{q}^{\mathrm{HP}}$ & $5.21 \pm 0.07$ & $3.53 \pm 0.09$ & $2.86 \pm 0.09$ & $2.42 \pm 0.09$ & $1.98 \pm 0.07$ & $1.744 \pm 0.02$ \\
$\lambda_{s}^{\mathrm{HP}}$ & $1.565^{*}$ & $1.39 \pm 0.05$ & $1.45 \pm 0.05$ & $1.34 \pm 0.06$ & $1.25 \pm 0.18$ & $1.155 \pm 0.03$ \\
$\gamma_{q}^{\mathrm{HP}}$ & $0.366 \pm 0.008$ & $0.49 \pm 0.03$ & $1.54 \pm 0.37$ & $1.66 \pm 0.14$ & $1.65 \pm 0.01$ & $1.64 \pm 0.01$ \\
$\gamma_{s}^{\mathrm{HP}}$ & $0.216 \pm 0.009$ & $0.40 \pm 0.03$ & $1.61 \pm 0.07$ & $1.62 \pm 0.25$ & $1.52 \pm 0.06$ & $1.63 \pm 0.02$ \\
$\lambda_{I 3}^{\mathrm{HP}}$ & $0.875 \pm 0.166$ & $0.877 \pm 0.05$ & $0.935 \pm 0.013$ & $0.960 \pm 0.027$ & $0.973 \pm 0.014$ & $0.975 \pm 0.005$ \\
\hline$\mu_{\mathrm{B}}[\mathrm{MeV}]$ & 759 & 574 & 390 & 347 & 276 & 227 \\
$\mu_{\mathrm{S}}[\mathrm{MeV}]$ & 180 & 141 & 83.7 & 77.6 & 62.0 & 56.0 \\
\hline
\end{tabular}

hadron masses. In order to relate the initial values $\gamma_{i}^{\mathrm{HP}}\left(t_{f}\right), i=q, s$, at freeze-out (subscript- $f$ ), to the QGP fireball source value $\gamma_{i}^{\mathrm{QGP}}\left(t_{f}\right), i=q$, s, we match across phase boundary by considering the continuity of entropy and strangeness. In principle, only the occupancies are discontinuous at QGP hadronization, the other SH parameters are smooth. For this reason, we attached phaseindices 'QGP' and 'HP' only to occupancy parameters, and when these are omitted we always mean implicitly the confined hadron phase HP.

\section{Hadron Yields: Data and Fits}

A complete analysis of experimental hadron yield results requires a significant book-keeping and fitting effort in order to allow for resonances, particle widths, full decay trees, isospin multiplet sub-states. A program SHARE (Statistical HAdronization with REsonances) suitable to perform this data analysis is available for public use $[12,13]$. This program implements the PDG [14] confirmed (4-star) set of particles and resonances, and we use [18] the recent determination of $\sigma$ meson mass [19] $\left(m_{\sigma}=484, \Gamma_{\sigma} / 2=255 \mathrm{MeV}\right)$. The data set we use in table 1 is the latest NA492008 results [20], together with, as reference, AGS data set at their highest energy, see [21, 18]. A star at $\lambda_{s}$ indicates that we fixed the value by strangeness conservation, which we do at AGS considering a small data set. 
Table 2: Predictions: AGS/SPS particle yields including NA49 2008 data, all SHARE 2.2.

\begin{tabular}{|c|c|c|c|c|c|c|}
\hline$E[A \mathrm{GeV}]$ & 11.6 & 20 & 30 & 40 & 80 & 158 \\
\hline$\sqrt{s_{\mathrm{NN}}}[\mathrm{GeV}]$ & 4.84 & 6.26 & 7.61 & 8.76 & 12.32 & 17.27 \\
\hline$y_{\mathrm{CM}}$ & 1.6 & 1.88 & 2.08 & 2.22 & 2.57 & 2.91 \\
\hline$N_{4 \pi} /$ centr. & m.c. & $7 \%$ & $7 \%$ & $7 \%$ & $7 \%$ & $5 \%$ \\
\hline$b \equiv B-\bar{B}$ & 375.6 & 348.1 & 348.6 & 349.9 & 349.5 & 361.7 \\
\hline$(s-\bar{s}) /(s+\bar{s})$ & 0 & -0.119 & -0.037 & -0.007 & -0.017 & -0.064 \\
\hline$\pi^{+}$ & 134.0 & 189.9 & 243.3 & 292.5 & 434.7 & 617.2 \\
\hline$\pi^{-}$ & 161.2 & 223.4 & 278.5 & 324.1 & 469.6 & 663.7 \\
\hline $\mathrm{K}^{+}$ & 17.5 & 41.1 & 50.2 & 53.4 & 72.7 & 111.3 \\
\hline $\mathrm{K}^{-}$ & 3.60 & 10.3 & 15.9 & 19.7 & 33.4 & 54.7 \\
\hline $\mathrm{K}_{\mathrm{S}}$ & 10.9 & 26.3 & 33.1 & 36.2 & 52.1 & 81.3 \\
\hline$\phi$ & 0.47 & 1.82 & 2.10 & 2.64 & 4.23 & 7.37 \\
\hline$p$ & 173.2 & 162.9 & 166.2 & 137.2 & 138.6 & 145.9 \\
\hline $\bar{p}$ & 0.022 & 0.207 & 0.57 & 0.74 & 2.46 & 5.39 \\
\hline$\Lambda$ & 18.7 & 29.3 & 39.5 & 36.9 & 41.3 & 48.5 \\
\hline $\bar{\Lambda}$ & 0.016 & 0.16 & 0.40 & 0.62 & 1.77 & 4.02 \\
\hline$\Xi^{-}$ & 0.49 & 1.34 & 2.45 & 2.77 & 3.42 & 4.55 \\
\hline $\bar{\Xi}^{+}$ & 0.0026 & 0.028 & 0.065 & 0.145 & 0.35 & 0.82 \\
\hline$\Omega$ & 0.014 & 0.065 & 0.14 & 0.178 & 0.26 & 0.39 \\
\hline $\bar{\Omega}$ & 0.0008 & 0.0089 & 0.014 & 0.031 & 0.067 & 0.16 \\
\hline$\eta$ & 8.50 & 16.7 & 19.5 & 23.2 & 36.2 & 55.6 \\
\hline$\eta^{\prime}$ & 0.43 & 1.13 & 1.06 & 1.40 & 2.34 & 3.78 \\
\hline$\rho^{0}$ & 11.2 & 19.0 & 13.1 & 18.9 & 30.6 & 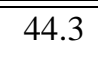 \\
\hline$\omega(782)$ & 5.94 & 12.9 & 11.1 & 14.9 & 25.7 & 38.4 \\
\hline$f_{0}(980)$ & 0.54 & 1.15 & 0.85 & 1.21 & 2.14 & 3.21 \\
\hline $\mathrm{K}^{0}(892)$ & 5.72 & 12.3 & 9.84 & 11.9 & 17.4 & 26.8 \\
\hline$\Delta^{0}$ & 37.9 & 33.1 & 25.16 & 26.3 & 26.9 & 28.0 \\
\hline$\Delta^{++}$ & 29.7 & 26.08 & 22.18 & 24.4 & 25.7 & 26.7 \\
\hline$\Lambda(1520)$ & 1.33 & 2.0 & 1.74 & 2.11 & 2.52 & 2.99 \\
\hline$\Sigma^{-}(1385)$ & 2.02 & 3.88 & 4.11 & 4.51 & 5.09 & 5.99 \\
\hline$\Xi^{0}(1530)$ & 0.16 & 0.43 & 0.69 & 0.84 & 1.08 & 1.45 \\
\hline
\end{tabular}

Aside of directly measured yields, we fit the baryon content ('measured' in terms of event centrality choice), the charge per baryon ('independently' measured to be the proton content in nuclei) and the strangeness balance, all three shown in separate top data section of the table. Since 'strangeness content' $s+\bar{s}$ is large, we choose to consider $\delta s=(s-\bar{s}) /(s+\bar{s})$. The errors we present for these entries are our estimates of how the error in measurement propagates into the statistical parameters.

A complete set of particle yields corresponding to the best parameters obtained at different reaction energies is presented in table 2. Below the stable particle yields, we show, in bottom section, the resonance yields which are not the measurable yields, but the initial values required in the study of a further resonance evolution [22]. The stable hadron yields agree well with the 

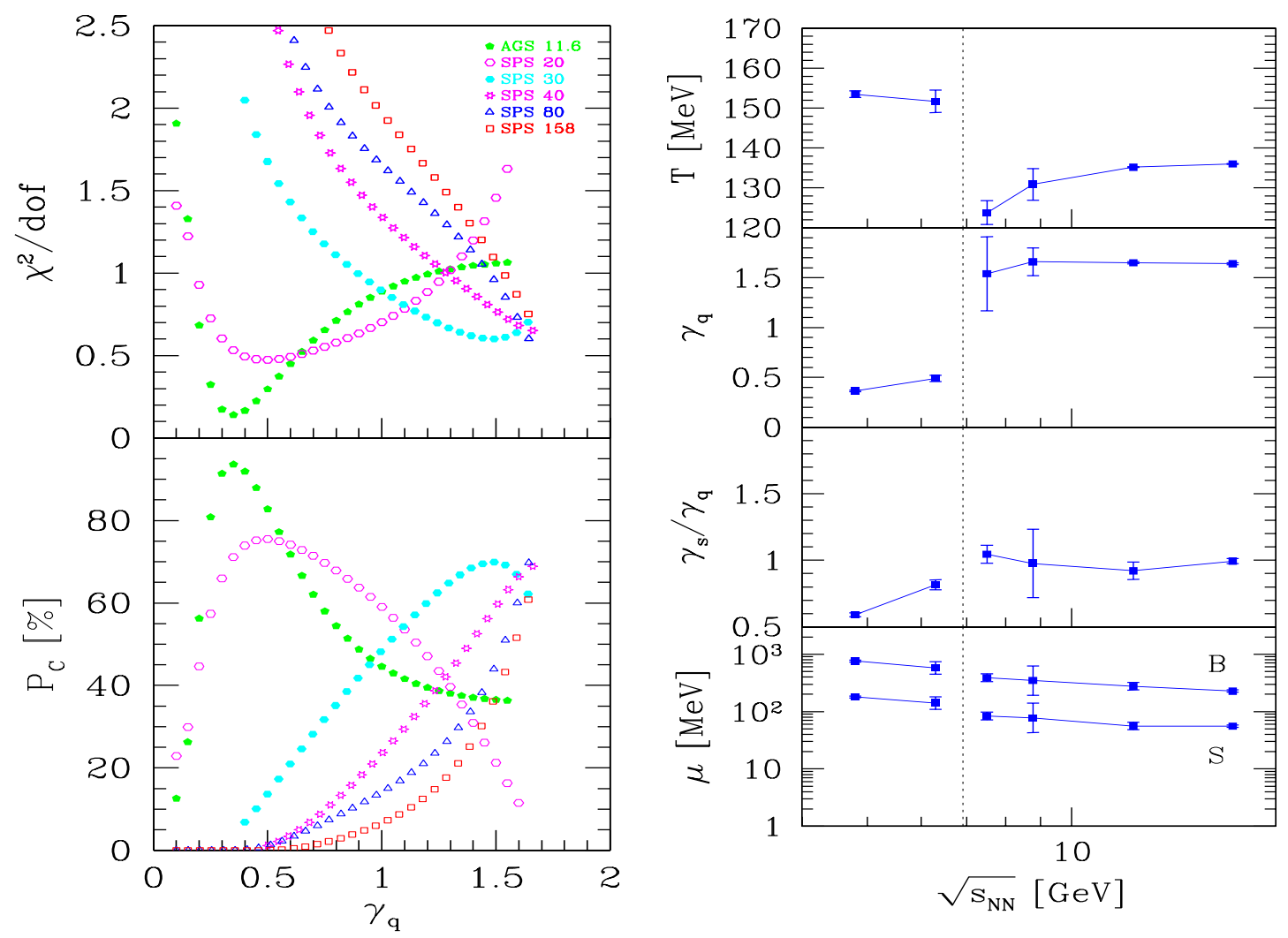

Figure 2: On left: $\chi^{2} /$ dof (top) and the associated significance level $P_{\mathrm{C}}[\%]$ (bottom) as function of fixed $\gamma_{q}$, for the AGS/SPS energy range. On right: the statistical parameters resulting from a fit at fixed $\gamma_{q}$, as function of reaction energy.

experimental energy dependence of the yield data well, this is, in particular, also the case for the $\phi$-yield. However, we expect a growing yield of $\Lambda$, which experimentally saturates at $80 A \mathrm{GeV}$ and then taking the center of measurements point, perhaps even decreases at $158 \mathrm{AGeV}$ compared to $80 \mathrm{AGeV}$.

We believe that imposing strangeness conservation can over constrain the fit, a step we only take if the data sample is small so that there is no sensitivity to the $\delta s=0$ constraint, as is the case at AGS. The values we find for $\delta s$ at SPS are all negative, see values stated in top of table 2 . The persistence of $\delta s<0$, if not result of some experimental NA49 bias, should be taken as indirect evidence for some new physics. We are looking forward to RHIC low energy run to resolve this important question. Note that the particle yields presented in table 2 are evaluated with the value of $\delta s$ as found in fit to be the best value. All yields shown in this table are prior to weak decays after hadronization.

We make, here, an effort to determine if the SH model we use makes good sense and if the parameters we employ are necessary. For this purpose, we show on left in figure 2 in the top frame the profile of $\chi^{2} /$ dof as function of $\gamma_{q}$, and in bottom frame, the profile of the confidence level $P_{\mathrm{C}}\left(\chi^{2}\right.$, dof $)$. We note that there is significant variation as function of $\gamma_{q}$ and that the best fit is not associated with the value $\gamma_{q}=1$. This shows that this parameter not used by many other 
groups $[11,15,16,17]$ is neither redundant nor can it assume a fixed value, tacitly set to $\gamma_{q}=1$, associated with thermal equilibrium ratio of baryons to mesons. (note that in the following, we always refer to $\gamma_{q}^{\mathrm{HP}}$ and thus we sometimes omit as in this paragraph the superscript).

We see, on left in figure 2, that our fit results separate into two groups. The AGS data point and the lowest energy SPS data point have a clear preference for $\gamma_{q}^{\mathrm{HP}}<1$. This suggests that at these two reaction energies (11.6 and $20 \mathrm{~A} \mathrm{GeV})$ the yield of hadrons and in particular of baryons are suppressed, where the benchmark production level is a chemically equilibrated hadron phase at the temperature $T \simeq 152 \pm 2 \mathrm{MeV}$. For the $30,40,80$ and $156 \mathrm{~A} \mathrm{GeV} \mathrm{SPS}$ reactions, we see that our fit results favor enhanced yields of hadrons and in particular that of baryons compared to the benchmark which is a rather low chemical freeze-out $T<140 \mathrm{MeV}$.

Sometimes it seems to us that other groups resist the use of $\gamma_{q}^{\mathrm{HP}}$ since they did not incorporate this important quantity consistently in their fit programs. Claims that SPS fits which include $\gamma_{q}$ are unstable were not true for SPS energy range and are certainly false after the final complete data set is now available. Aside of $\gamma_{q}$, there is another important nuance between our and 'their' fit. We do not see a value of $\lambda_{I 3}$ published by several other groups, thus we are not sure that this important quantity is employed in the fits, along with the requirement that net electrical charge of the system is that of the participating protons.

On right in figure 2, we show the fit results for statistical parameters, shown for each $\sqrt{s_{\mathrm{NN}}}$. We note that there is a rapid shift in behavior as we go across $\sqrt{s_{\mathrm{NN}}}=7 \mathrm{GeV}$ (vertical dotted line) of $T, \gamma_{q}$, and $\gamma_{q} / \gamma_{s}$, and of the volume $V$ seen in the table 1 . This contrasts with the much smoother behavior of the baryo chemical $\mu_{B}$ and strangeness $\mu_{S}$ potentials (bottom frame on right in figure 2) showing that across the entire reaction energy domain the chemical conditions change smoothly. On the other hand, there is considerable shift in chemical potentials $\mu_{i}$ of individual baryons and anti baryons at the boundary $\sqrt{s_{\mathrm{NN}}}=7 \mathrm{GeV}$. Individual $\mu_{i}$ are evaluated following the quark content, for example:

$$
\left.\left.\mu_{\Xi(s s q)}=2 T \ln \left(\gamma_{s}+\lambda_{s}\right)+T \ln \left(\gamma_{q}+\lambda_{q}\right)\right), \quad \mu_{\bar{\Xi}(\bar{s} \bar{s} \bar{q})}=2 T \ln \left(\gamma_{s}-\lambda_{s}\right)+T \ln \left(\gamma_{q}-\lambda_{q}\right)\right) .
$$

In figure 3 on left, we see that considerable discontinuity arises for all baryon chemical potentials between these two domains. The energy needed to add a baryon is thus smooth, while the energy to add any individual particle carrying baryon number is not.

In figure 3 on right, we compare our fit of $\mathrm{K}^{+} / \pi^{+}$with data as function of $\sqrt{s_{\mathrm{NN}}}$. While our non-equilibrium model works very well and the peak of the horn we see in the data appears to be another consequence of the change in hadronization behavior near $\sqrt{s_{\mathrm{NN}}}=7 \mathrm{GeV}$, the chemical equilibrium model [11] (dotted, blue) does not have a priory this capability. We believe that in order to explain the NA49 results one needs:

a) the strangeness yield parameter $\gamma_{s}^{\mathrm{HP}}$;

b) and ability to choose baryon to meson ratio independent of temperature, that is $\gamma_{q}^{\mathrm{HP}} \neq 1$.

The above parameters could be de-facto achieved by a fine tuned spectrum of hadron resonances [10]. We note that the decreasing value of $\mathrm{K}^{+} / \pi^{+}$beyond the peak has recently been confirmed by the trial preparatory low energy run at RHIC [23].

In closing this discussion, we note that an analysis of high energy RHIC data lacks the important constraint to a fixed baryon content, since unlike at SPS the RHIC results are at central rapidity and the baryon content is one of variables which are outcome of the fit. The absence of baryon yield 

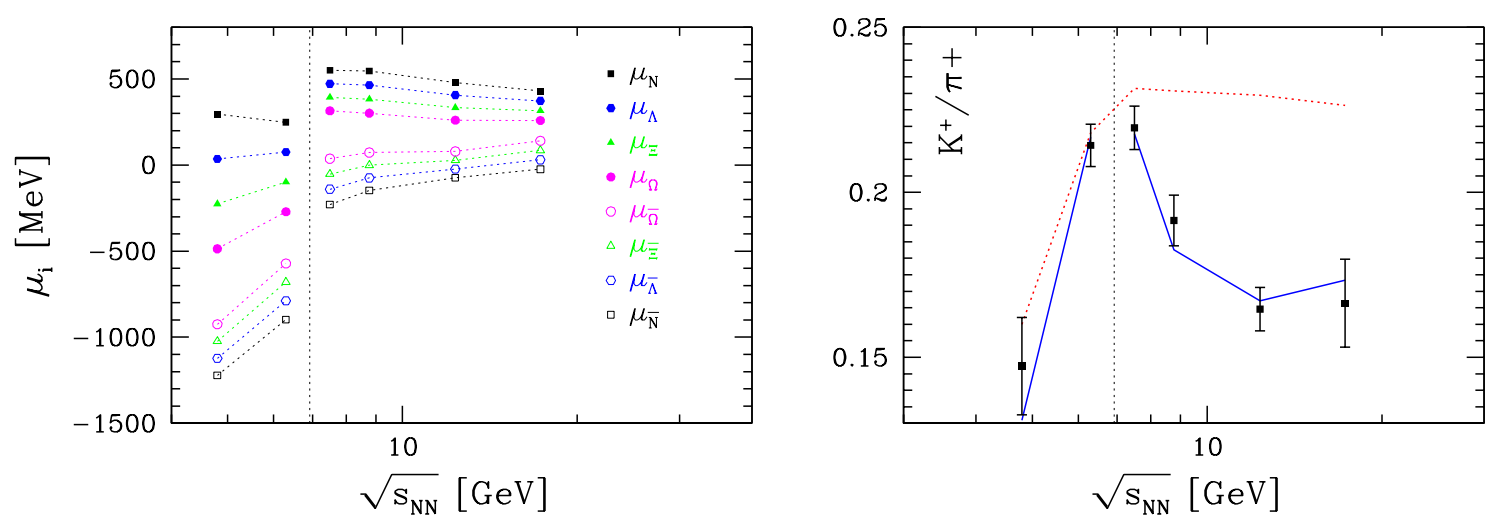

Figure 3: On left, the chemical potentials of all baryons and antibaryons in HP. On right, our model solid line (blue) tracks $\mathrm{K}^{+} / \pi^{+}$yield (data) as function of reaction energy. Dotted (red) line: equilibrium model [11].

constraint reduces the stability of the many-parameter fit and a different approach must be taken which we will discuss elsewhere.

\section{Discussion of data analysis}

Q: As I see you do not constrain net strangeness to zero - I think that this maybe the reason your effort in this field is not taken as seriously as it should!

A: Indeed, we have gained better insight in past 15 years regarding strangeness conservation, as compared to our older work [24]. At the time we argued that the slowness of the weak strangeness decay assures that in hadronic interactions net strangeness should be zero: $\Delta s=s-\bar{s}=0$. Today, we allow $\delta s$ to behave like a measurement, that is, we introduce it with an error $\delta s=0 \pm 0.05$. The reasoning is as follows

a) This is a test of the hypothesis that weak decays remains weak in QGP phase, and the net strangeness remains conserved;

b) NA49 did measure many, but not all particles carrying strangeness, e.g., $\Sigma^{ \pm}$has not been measured, this yield is uncertain and thus $\delta s=0$ cannot be tested to better than about 5\%;

c) Summing all measured and unmeasured hadrons in strangeness 'conservation' condition, $\Delta s=$ $\sum_{i} h_{s}^{i}-\sum_{j} h_{\bar{s}}^{j} \rightarrow 0$, combines independent measurement errors and thus, even if NA49 had measured all strangeness carrying hadrons, there would be a residual statistical error present in $\delta s-$ another way to understand this is to note that we cannot confirm that weak decays in QGP remain weak to better than the progressing error of individual contributing measurements;

d) Some strangeness could escape detection in unknown 'particles', for example being bound in (nearly) $u d s$-quark-symmetric semi-stable strangelett (a small drop of quark matter), this leads to $\delta s<0-$ which is what we find consistently in our fits.

Q: In what sense is $P_{\mathrm{C}}$ (see figure 2, left bottom frame) conveying confidence, and in 'what thing', in what way does this quantity deserve this name, and, I would think it is better to suppress this 
part of the figure as it contains redundant information!

A: You are familiar with the Section 32.3 in PDG [14] (PDG: particle data group biannual issue of 'Review of particle physics'), which provides further discussion of the elusive meaning of $P_{\mathrm{C}}$, and figure 32.2 shows which fixed values of $P_{\mathrm{C}}$ arise for given values $\chi^{2} /$ dof and dof. The SHARE program evaluates $P_{\mathrm{C}}$ for each fit. Abbreviating this section in PDG in a few words: When fitting a statistical data set to a model with a few parameters the value of $P_{\mathrm{C}}\left(\chi^{2}\right.$, dof) predicts (in sense of Bayesian inferment) the likelihood of repeat experiment to produce the same outcome for the model parameters. Thus, $P_{\mathrm{C}}$ expresses confidence in the validity of the model.

Effectively, $P_{\mathrm{C}}\left(\chi^{2}\right.$, dof $)$ also expresses confidence in the data, provided that we believe in the model: when we intentionally alter an experimental data point by 2 s.d., our data fit remains stable in the sense that we find nearly the same model parameters, but $P_{\mathrm{C}}$ becomes much smaller, and the one data point which is not well fitted is the one we altered. Note that $\chi^{2} /$ dof is not as sensitive to this consideration, since the effect of one 'wrong' measurement is diluted by the magnitude of dof. The function $P_{\mathrm{C}}\left(\chi^{2}\right.$, dof $)$ turns insensitive in the limit dof $\rightarrow \infty$, but we are far from this. In fact, in our study of the fits to the date, we found that the value of $P_{\mathrm{C}}$ is much more interesting when comparing for example NA49 data at different energies since the number of available data points varies and thus dof varies along with $\chi^{2}$. Thus, if we were to suppress a result it would be the profile of $\chi^{2} /$ dof, but that cannot be done since people want to see the value of $\chi^{2} /$ dof.

Q: I note that in your recent work [25] all 6 best values $P_{\mathrm{C}}$ are larger than 0.8 which is very unlikely to happen for 6 independent measurements.

A: Yes, you will further note that in this paper all NA49 fits carried out with the final 2008 data set converge to a common best value $P_{\mathrm{C}}=70 \%$. This value has higher $P_{\mathrm{C}}$ than one could expect on statistical grounds (50\%), but we note that we have treated systematic errors as if these were statistical errors, and thus effectively made errors too large. In one case, we also increased the error bar of NA49, beyond the statistical and systematic error, namely we doubled error for $\Lambda$-yield at $80 \mathrm{AGeV}$, and thus it is of same relative magnitude as the error at $158 \mathrm{AGeV}$. We did this since our fit and $P_{\mathrm{C}}$ indicated that this one NA49 value was out of systematics of other particles measured at this energy. We fit this measurement but with an error which is modified as stated above.

Q: How did the SPS heavy ion energy scan research program come to be?

A: In early 1980's there were several studies of the mechanism governing the development of deconfinement as function of reaction energy. Looking at this work one finds energy estimates for transition to QGP within a range well beyond the SPS reach. Only a more persistent search will unearth a minority view, for example in the opening of conclusions of Ref.[26] we read: "The formation of a baryon-rich quark-gluon plasma appears to be an important reaction channel in collisions of heavy nuclei in the energy region of $2.5-5 \mathrm{GeV}$ per nucleon in the center of mass frame of reference". We recall that the $\mathrm{K}^{+} / \pi^{+}$peak is at $2 \times 3.5 \mathrm{GeV}$, right in the middle of this prediction, obtained considering the growth of 'QGP seeds', small deconfined drops of matter. Strange particle production signature of this transformation was the favorite experimental approach [27]. However, only when it was proposed that an easily accessible observable $\mathrm{K}^{+} / \pi^{+}$could suffice [28], the research program to scan the hadron production as function of reaction energy in the SPS domain took off. This development is consistent with the CERN announcement of February 7, 2000 that a "new state of matter" is produced in the top SPS energy central $\mathrm{Pb}-\mathrm{Pb}$ collisions. 


\section{Physical Properties of the Source}

The SHARE program remains till this day a unique tool when it comes to the evaluation of the physical properties of the source of hadrons. Once a fit is achieved, this is a straightforward task, however, with a significant bookkeeping challenge: each hadronic particle, stable, or resonance, given the statistical parameters $T, \mu, \ldots$, contributes to the physical properties such as pressure $P$, entropy density $S / V$, energy density $E / V$, and baryons also to net baryon density $b / V$, while all strange hadrons with s-contents contribute to $s / V$. These properties are of considerable importance in discussion of the meaning of the fit to the data.

We believe that the extensive properties such as $P$, or $s$ are more reliable than the intensive statistical model parameters. The probability $P_{\mathrm{C}}$ is in fact indicating the reliability of finding in a redo of experiment and analysis in framework of the same physical model the same $P, S, E, b, s$. This is so since some elements of our model are not fully established and thus will evolve in time. For example, the hadron spectrum will be in the coming decade better understood and this may alter some statistical properties. However, the occupancy parameters $\gamma_{i}$ compensate to a large extent just this model dependence, and thus a refinement of hadron mass spectrum should result in some change of $\gamma_{i}$, but, as we hope, little changed evaluation of the physical properties of the fireball. Said differently, for example, the observed pion yield is a good measure of the entropy produced with little model dependence.

A sample of our findings for the physical properties is presented, in figure 4 , as ratio of different extensive quantities: on left, we see $s / b, s / S$, and $E / s$; on right top frame, the pressure $P$, and bottom right, the thermal energy $E$ per primary hadron $h_{\mathrm{p}}$. The results we find are the symbols, the lines guide the eye. All solid (blue) symbols and lines are full non-equilibrium model. On right the open, dashed (red) triangles and lines are semi-equilibrium model with $\gamma_{q}^{\mathrm{HP}}=1$. Since $\gamma_{q}^{\mathrm{HP}}$ is artificially fixed, the pressure is not the same in both models, and, there is a great reduction of confidence level $P_{\mathrm{C}}$ in this result, as is seen in figure 2, left bottom frame: compare highest value of $P_{\mathrm{C}}$ with that arising at a fixed $\gamma_{q}^{\mathrm{HP}}=1$. Said differently, the values we present on right in figure 4

for $\gamma_{q}^{\mathrm{HP}}=1$ (red, dashed) and $\gamma_{q}^{\mathrm{HP}}$ fitted (solid, blue) are different since these are in principle two different models to be distinguished by the quality of the fit.

Perhaps the most interesting finding is seen in figure 4 on right in the top frame: the hadronization pressure is practically constant, near about $82 \mathrm{MeV} / \mathrm{fm}^{3}$ (non equilibrium model, solid line), at highest three SPS energies. The absence of fluctuations in this result is most remarkable, a smooth line is connecting 40,80 and $158 \mathrm{AGeV}$ result. The preliminary NA49 data gave less 'constant' result $[25,18]$.

The baryon content of the source fireball is fixed by the choice of centrality, thus the rise of $s / b$ (top left) indicates that strangeness production increases rapidly, and smoothly, with reaction energy. Note that strangeness content increases six fold between 10 and $158 \mathrm{AGeV}$ reaction energy, and by a factor 2.5 between 30 and $158 \mathrm{AGeV}$. On the other hand, the value range $s / S \in(0.019,0.022)$ combined with the growth of $s / b$ shows that the fireball experiences, as function of reaction energy, a growth in both entropy and strangeness at a comparable rate.

The high value of $s / S$ is established much earlier in time, in a much more dense phase than the chemical freeze-out. The behavior of $s / S$ suggests that at $40 \mathrm{AGeV}$ (and perhaps event at 30 $A \mathrm{GeV}$ ), in an early stage of the fireball when $s$ and $S$ is made, the same quark-gluon degrees of 

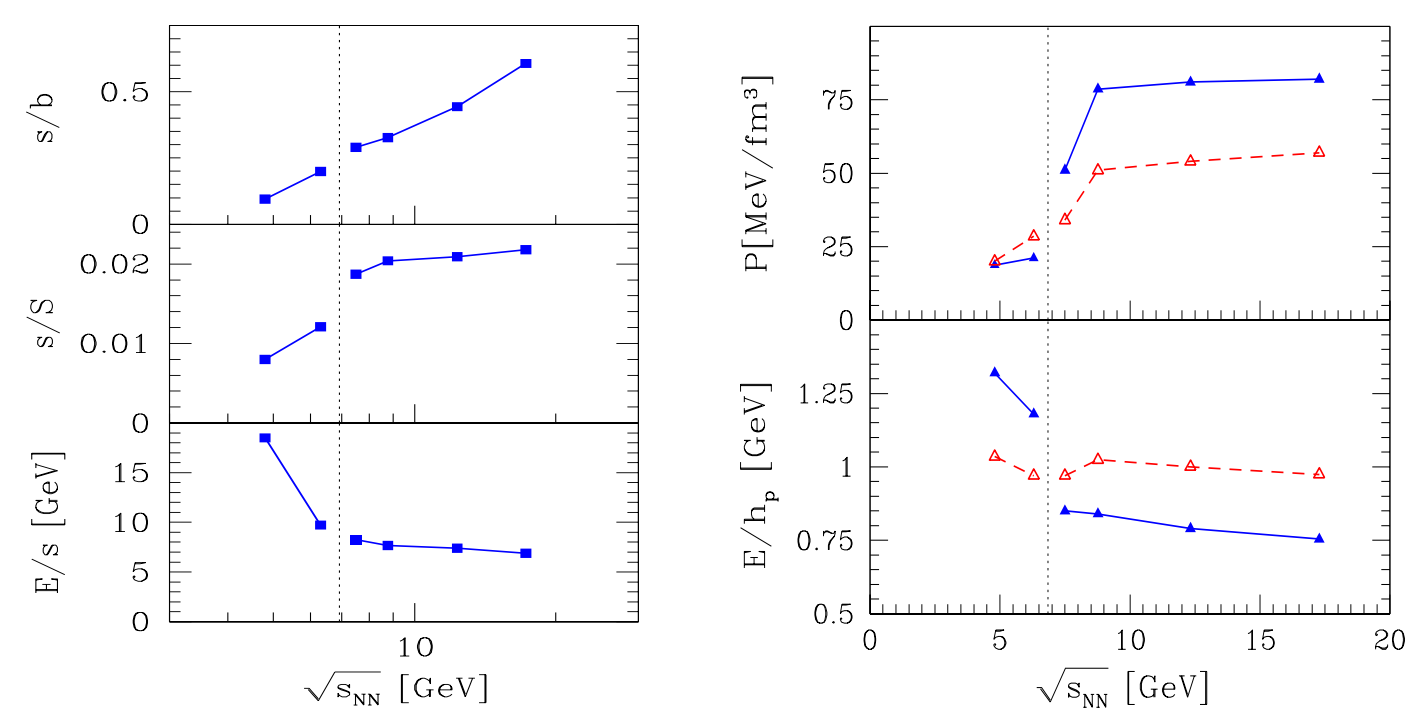

Figure 4: Physical properties of the hadronizing fireball. On left $4 s / b, s / S, E / s$ and in right pressure $P$ and thermal energy per primary hadron $E / h_{p}$. The red lines on right correspond to $\gamma_{q}^{\mathrm{HP}}=1$ and have a much lower confidence level, $P_{C}$, see figure 2 .

freedom are already active. Consideration of kinetic theory [29] suggest that the growth of $s / S$ is due to a gradual as function of reaction energy approach to chemical equilibrium, first by gluons, than by light quarks, and later by strange quarks. We refer to a more thorough discussion of the importance of observable $s / S$ presented elsewhere [30,31].

The cost in global thermal energy $E$ to make a strange quark pair yield $s$, including those bound as pairs in $\eta, \eta^{\prime}$ and $\phi$ is seen on left, in the bottom frame of figure 4 . This value is as low as $6 \mathrm{GeV}$ at highest SPS energy. Here, $E$ is the thermal energy content of the hadron system and $s$ is as before the absolute yield of strange quark pairs. A strange quark pair at hadronization has an energy near to $0.9 \mathrm{GeV}$. The remainder of the energy content per this pair, in a nearly equilibrated deconfined source of hadrons, is contained in the thermal light quarks and gluons. This argument accounts well for the remaining $5 \mathrm{GeV}$. The greater energy required to produce strangeness pair at the lower reaction energies is consistent with the hypothesis that, prior to hadronization, strangeness in the fireball is far below chemical equilibrium, as is seen in the small $s / b$ ratio.

Finally, the bottom frame on right considers the hypothesis that the chemical freeze-out condition is related to energy required for making a primary hadron [16]. We see that within the semi-equilibrium model (red, dashed line), the value is indeed, within a reasonable margin, oscillating near $E / h_{p} \simeq 1 \mathrm{GeV}$. However, the high confidence result (blue, solid line) within the non equilibrium model demonstrates a strong variation of $E / h_{p}$ which drops from above $1.3 \mathrm{GeV}$ to $0.75 \mathrm{GeV}$, and thus, $E / h_{p}$ is not a good criteria to define freeze-out of hadrons. Moreover, this variable has a priory no fundamental meaning, and this quantity depends strongly on the spectrum of hadrons used, equivalently, the value of $\gamma_{q}$. 


\section{Discussion and Conclusions}

For low reaction energies (AGS 10.6 AGeV and SPS $20 A \mathrm{GeV}$ ), we find hadrons originate from a relatively large $\left(V>3500 \mathrm{fm}^{3}\right)$, dilute $\left(\gamma_{q}<0.5\right)$, yet relatively hot system $(T>150 \mathrm{MeV})$. The strangeness abundance is low and fast growing. At higher reaction energies (SPS 30, 40, 80 and $158 \mathrm{AGeV}$ ), the volume per hadron is less than half as large, and the chemical freeze-out proceeds at about $20 \%$ lower $T$. We know from the experimental results that there is a rapid expansion of the dense matter. We conclude that in the buildup of the matter flow there was significant cooling of the fireball matter. Particle spectra confirm that there is fast flow in this SPS energy range. Our hypothesis, which is consistent with all results described, is that we are, already at SPS, seeing sudden hadronization of a supercooled deconfined phase [32] at 40, 80 and $158 \mathrm{AGeV}$ reaction energy, and to some lesser extend at $30 \mathrm{AGeV}$. This hypothesis allows to qualitatively explain the high $\gamma_{q}^{\mathrm{HP}}>1$ value, and is consistent with physical properties of the system we observe implicitly by measuring the hadronization parameters.

There are several possible explanations of the AGS $10.6 \mathrm{AGeV}$ and SPS $20 A \mathrm{GeV}$ low energy behavior:

a) the most straightforward alternative is the usual confined, hadron phase in which antibaryon annihilation, which reduces the baryon to meson ratio, takes place, which leads to chemically under saturated $\gamma_{q}<1$;

b) given the large baryo-chemical potential (see bottom of table1) the freeze-out is in a domain in which a complicated QCD phase structure is expected and the physical properties we find are consistent with breakup of a valance quark deconfined matter [18]. Such a phase has relatively massive constituents $\left(m_{q} \simeq 330, m_{s} \simeq 500 \mathrm{MeV}\right)$ chiral symmetry is strongly broken, even though deconfinement prevails - only for $\mu_{i} \rightarrow 0$ there is empirical and lattice evidence that chiral symmetry and deconfinement are restored at the same condition. The matter flow of a heavy constituent matter is expected to be slow, and thus, hadronization mechanism differs profoundly from what one sees at higher energy;

c) Considering relatively high $P_{\mathrm{C}}$ near $\gamma_{q}=1$, for $10.6 \mathrm{AGeV}$ we have $45 \%$ and for $20 \mathrm{AGeV}$ we have $60 \%$, we could simply discount our results at low energies forcing chemical equilibrium $\gamma_{q}=1$ for light quarks, claiming that the (small) best fit values of $\gamma_{q}$ are an artifact of experimental data points. To some extent this remark can be made also for $30 \mathrm{AGeV}$ data, but cannot be made for 40, 80 and $158 \mathrm{AGeV}$.

Perhaps the most intriguing result of this analysis is the smoothness, and even near constancy, of physical properties of the fireball at chemical freeze-out condition for the top three SPS energies 40, 80 and $158 \mathrm{AGeV}$. Of particular physical interest is the value of hadronization pressure $P \simeq 82$ $\mathrm{MeV} / \mathrm{fm}^{3}$, which is nearly constant. In a phase transformation from quarks to hadrons, the pressure of quarks is transferred into the pressure of color-neutral hadrons, which can escape from the deconfined fireball. Since the flow pressure of quarks transfers smoothly into that of hadrons, we conclude that the thermal pressure of produced hadrons, $P \simeq 82 \mathrm{MeV} / \mathrm{fm}^{3}$, provides a first estimate of the pressure of the vacuum which keeps color charged quarks inside the fireball up to the point of sudden fireball break-up.

To conclude, we find analyzing the final NA49 SPS data that the most central $\mathrm{Pb}-\mathrm{Pb}$ collisions at 40,80 and $158 \mathrm{AGeV}$, corresponding to $\sqrt{s_{\mathrm{NN}}}=8.76,12.32$, and $17.27 \mathrm{GeV}$, are showing very 
similar behavior at hadronization, where the physical and statistical properties of the source can be interpreted in terms of a sudden QGP fireball decomposition. The SPS reaction at $30 \mathrm{AGeV}$, corresponding to $\sqrt{s_{\mathrm{NN}}}=7.61 \mathrm{GeV}$, is an intermediate case, which in many aspects is similar to the higher energies, but the hadronization pressure evaluated in the ensemble of reaction events is significantly reduced. The reactions at AGS 10.6 and SPS $20 \mathrm{AGeV}$, corresponding to $\sqrt{s_{\mathrm{NN}}}=4.84$ and $6.26 \mathrm{GeV}$, are in a different class which cannot be yet fully categorized, and require more precise and comprehensive experimental data in order to allow a more specific conclusion about the applicable reaction mechanism.

\section{Acknowledgments}

We thank Marek Gaździcki for his interest and valuable comments. Work supported by a grant from: the U.S. Department of Energy DE-FG02-04ER4131 and by DFG, LMUexcellent. LPTHE, Univ. Paris 6 et 7 is: Unité mixte de Recherche du CNRS, UMR7589

\section{References}

[1] J. Rafelski and R. Hagedorn, "From Hadron Gas To Quark Matter. 2", in Statistical Mechanics of Quarks and Hadrons, H. Satz, ed. (Norht Holland 1980) pp. 253-272, also: Preprint CERN-TH-2969, October 1980. A scan of this articles is also available at http://www.physics.arizona.edu/ rafelski/rare.htm.

[2] B. Muller, "The Physics Of The Quark - Gluon Plasma", Lecture Notes in Physics, v.225, 1985.

[3] P. Koch, B. Muller and J. Rafelski, Phys. Rept. 142 (1986) 167.

[4] R. J. Fries, B. Muller, C. Nonaka and S. A. Bass, Phys. Rev. Lett. 90 (2003) 202303 [arXiv:nucl-th/0301087]; and Phys. Rev. C 68 (2003) 044902 [arXiv:nucl-th/0306027]. and Kang Seog Lee, Steffen Bass, Berndt Mueller, and Chiho Nonaka "Hadronization via Recombination", arXiv:0812.4724v1 [nucl-th].

[5] H. Z. Huang and J. Rafelski, AIP Conf. Proc. 756, 210 (2005) [arXiv:hep-ph/0501187]; S. S. Adler et al. [PHENIX Collab.], Phys. Rev. C 69 (2004) 034909, 034910 [arXiv:nucl-ex/0307022]; L. J. Ruan [STAR Collab.], J. Phys. G 31 (2005) S1029 [arXiv:nucl-ex/0503015] and references therein.

[6] B. Andersson, G. Gustafson and H. Pi, Z. Phys. C 57 (1993) 485.

[7] A. Laszlo [NA49 Collaboration], "Nuclear modification at $\sqrt{s_{N N}}=17.3 \mathrm{GeV}$, measured at NA49", arXiv:0805.4771 [nucl-ex].

[8] W. Broniowski, M. Chojnacki, W. Florkowski and A. Kisiel, Phys. Rev. Lett. 101 (2008) 022301 [arXiv:0801.4361 [nucl-th]], and references therein.

[9] G. Torrieri and J. Rafelski, New J. Phys. 3, 12 (2001) [arXiv:hep-ph/0012102], and references therein.

[10] A. Andronic, P. Braun-Munzinger and J. Stachel, arXiv:0812.1186 [nucl-th].

[11] P. Braun-Munzinger, et al. Phys. Lett. B 344 (1995) 43; ibid 365 (1996) 1; Phys. Lett. B ibid 465 (1999) 15; ibid 518 (2001) 41.

[12] G. Torrieri, S. Steinke, W. Broniowski, W. Florkowski, J. Letessier and J. Rafelski, Comput. Phys. Commun. 167 (2005) 229 [arXiv:nucl-th/0404083]. 
[13] G. Torrieri, S. Jeon, J. Letessier and J. Rafelski, Comput. Phys. Commun. 175 (2006) 635 [arXiv:nucl-th/0603026].

[14] W. M. Yao et al. [Particle Data Group], J. Phys. G 33 (2006) 1.

[15] F. Becattini, J. Manninen and M. Gazdzicki, Phys. Rev. C 73 (2006) 044905 [arXiv:hep-ph/0511092].

[16] J. Cleymans, H. Oeschler, K. Redlich and S. Wheaton, Phys. Rev. C 73 (2006) 034905 [arXiv:hep-ph/0511094].

[17] I. Kraus, J. Cleymans, H. Oeschler, K. Redlich and S. Wheaton, Phys. Rev. C 76 (2007) 064903 [arXiv:0707.3879 [hep-ph]].

[18] J. Letessier and J. Rafelski, Eur. Phys. J. A 35 (2008) 221 [arXiv:nucl-th/0504028].

[19] R. Garcia-Martin, J. R. Pelaez and F. J. Yndurain, Phys. Rev. D 76 (2007) 074034 [arXiv:hep-ph/0701025]; R. Kaminski, R. Garcia-Martin, P. Grynkiewicz and J. R. Pelaez, "Sigma pole position and errors of a once and twice subtracted dispersive analysis of pi-pi scattering data," arXiv:0811.4510 [hep-ph].

[20] C. Alt et al. [NA49 Collab.], Phys. Rev. C 78 (2008) 044907 [arXiv:0806.1937 [nucl-ex]]; Phys. Rev. C 78 (2008) 034918 [arXiv:0804.3770 [nucl-ex]]; Phys. Rev. C 77 (2008) 024903 [arXiv:0710.0118 [nucl-ex]]; Phys. Rev. Lett. 94 (2005) 192301 [arXiv:nucl-ex/0409004]; T. Anticic et al. [NA49 Collab.], Phys. Rev. Lett. 93 (2004) 022302 [arXiv:nucl-ex/0311024]; S. V. Afanasiev et al. [The NA49 Collab.], Phys. Rev. C 66 (2002) 054902 [arXiv:nucl-ex/0205002].

[21] J. Letessier, J. Rafelski and G. Torrieri, "Deconfinement energy threshold: Analysis of hadron yields at 11.6-A-GeV," arXiv:nucl-th/0411047, see also Ref.[18].

[22] I. Kuznetsova and J. Rafelski, arXiv:0811.1409 [nucl-th], Phys. Rev. C in press (2009); and Phys. Lett. B 668 (2008) 105 [arXiv:0804.3352 [nucl-th]].

[23] L. Kumar and f. t. S. Collaboration, "First results from Au+Au collisions at $\sqrt{\left(s_{N N}\right)}=9.2 \mathrm{GeV}$ in STAR," arXiv:0812.4099 [nucl-ex].

[24] J. Letessier, A. Tounsi, U. W. Heinz, J. Sollfrank and J. Rafelski, Phys. Rev. D 51 (1995) 3408 [arXiv:hep-ph/9212210].

[25] J. Rafelski, I. Kuznetsova and J. Letessier, J. Phys. G 35 (2008) 044011 [arXiv:0801.0588 [nucl-th]].

[26] M. Danos and J. Rafelski, "Baryon-rich quark-gluon plasma in nuclear collisions,", UCT-TP 7/84, Nov 1984, reprinted in Heavy Ion Phys. 14, 97 (2001) and archived at [arXiv:nucl-th/0011049], see also: J. Rafelski and M. Danos, "Perspectives In High-Energy Nuclear Collisions," NBSIR-83-2725, GSI-83-6, Jun 1983. 80pp., scan at http://www-lib.kek.jp/cgi-bin/img_index?200031578, and J. Rafelski and M. Danos, "Nuclear Matter Under Extreme Conditions,", UCT-TP 8/84, Nov 1984. 93pp. scan at http://www-lib.kek.jp/cgi-bin/img_index?200036132, in Hadrons and heavy ions, W.D. Heiss, edt., Springer Lecture Notes in Physics, 231 (1985).

[27] J. Rafelski, J. Letessier and A. Tounsi, Acta Phys. Polon. B 27 (1996) 1037 [arXiv:nucl-th/0209080].

[28] M. Gazdzicki and M. I. Gorenstein, Acta Phys. Polon. B 30, 2705 (1999) [arXiv:hep-ph/9803462].

[29] J. Alam, B. Sinha and S. Raha, Phys. Rev. Lett. 73 (1994) 1895.

[30] J. Letessier and J. Rafelski, Phys. Rev. C 75 (2007) 014905 [arXiv:nucl-th/0602047].

[31] I. Kuznetsova and J. Rafelski, Eur. Phys. J. C 51 (2007) 113 [arXiv:hep-ph/0607203].

[32] J. Rafelski and J. Letessier, Phys. Rev. Lett. 85 (2000) 4695 [arXiv:hep-ph/0006200]. 